\title{
Factors Influencing Survival Outcomes in Patients with Left Ventricular Dysfunction after Coronary Revascularization
}

\author{
Rama Kishore Yalampati ${ }^{1} \quad$ Lalitha N$^{1} \quad$ Naveen Kumar Murki ${ }^{1}$ \\ ${ }^{1}$ Department of Cardiology, NIMS, Hyderabad, Telangana, India \\ Address for correspondence Rama Kishore Yalampati, DM, \\ Department of Cardiology, NIMS, Hyderabad, Telangana, 500 082, \\ India (e-mail: honeyyrk@gmail.com).
}

Ind J Car Dis Wom 2021;6:27-32.

\begin{abstract}
Keywords

- left ventricular dysfunction

- coronary artery disease

- percutaneous transluminal coronary angioplasty

- coronary artery bypass grafting

Background Outcomes in patients with left ventricular (LV) dysfunction after coronary revascularization are influenced by multiple factors; however, it is difficult to compare a direct relationship between LV dysfunction and mortality due to the presence of confounding variables, so we attempted to observe the influence of risk factors on outcomes in severe LV dysfunction patients after revascularization and delineate if any of them have an effect on one-year mortality of such patients.

Methodology This is a single center prospective study, where the studied population were severe LV dysfunction patients who underwent percutaneous transluminal coronary angioplasty (PTCA) and followed-up for one year and at the end of one year, impact of gender, age, type 2 diabetes mellitus (DM), hypertension (HTN), obesity, chronic kidney disease (CKD), cerebrovascular accident (CVA), hypothyroidism, smoking and alcohol on one-year mortality and MACE (major adverse cardiovascular events), which included reinfarction (nonfatal), recurrence of angina, repeat percutaneous intervention $(\mathrm{PCI})$ or coronary artery bypass grafting (CABG) and heart failure. The above factors were analyzed and statistically approached to observe the impact of those risk factors on one-year mortality and determine whether the timing and mode of revascularization, and number of coronary arteries involved, had any influence on mortality or MACE events. By comparing different parameters of the study with respect to mortality, a regression analysis was made at the end of one year Results As many as 152 patients of severe LV dysfunction (ejection fraction < 30\%) were enrolled in the study, among which 115 (75.6\%) patients were males, and 37 patients were females $(24.3 \%)$, with a mean age of 57.6 years; 89 (58.5\%) patients were hypertensive, $80(52.6 \%)$ patients were diabetics, $42(27.6 \%)$ patients were smokers, 20 (13.1\%) patients were alcoholics, 9 (5.9\%) had CKD, one (0.6\%) patient had hypothyroidism and one (0.6\%) patient had a history of CVA; 46 (30.2\%) patients presented with acute coronary syndrome (ACS) and 106 (69.7) patients had chronic stable angina (CSA); 144 (94.7\%) patients underwent PTCA (percutaneous transluminal coronary angioplasty), while eight (5.2\%) patients underwent CABG. At the end of one year, mortality was six patients (3.94\%).

At the end of one year, regression analysis was done for all the confounding variables by observing their influence on the MACE or mortality; none of them showed statistically significant influence $(p>0.05)$.
\end{abstract}

published online

April 13, 2021
DOI https://doi.org/

$10.1055 / \mathrm{s}-0040-1709957$
(C)2021. Women in Cardiology and Related Sciences.

This is an open access article published by Thieme under the terms of the Creative Commons Attribution-NonDerivative-NonCommercial-License, permitting copying and reproduction so long as the original work is given appropriate credit. Contents may not be used for commercial purposes, or adapted, remixed, transformed or built upon. (https://creativecommons.org/licenses/by-nc-nd/4.0/).

Thieme Medical and Scientific Publishers Pvt. Ltd. A-12, 2nd Floor, Sector 2, Noida-201301 UP, India 
Conclusion At the end of one year, after revascularization in patients with severe LV dysfunction, no significant relationship could be ascertained between the mortality or MACE events and gender, age, DM, HTN, CKD, and alcohol or smoking. MACE or mortality may be attributed directly to LV dysfunction itself, and the observed mortality was higher than that of normal LV Function. Hence, in our study, LV dysfunction is the cause of mortality in the study population but not the other confounding variables.

\section{Abstract Image}

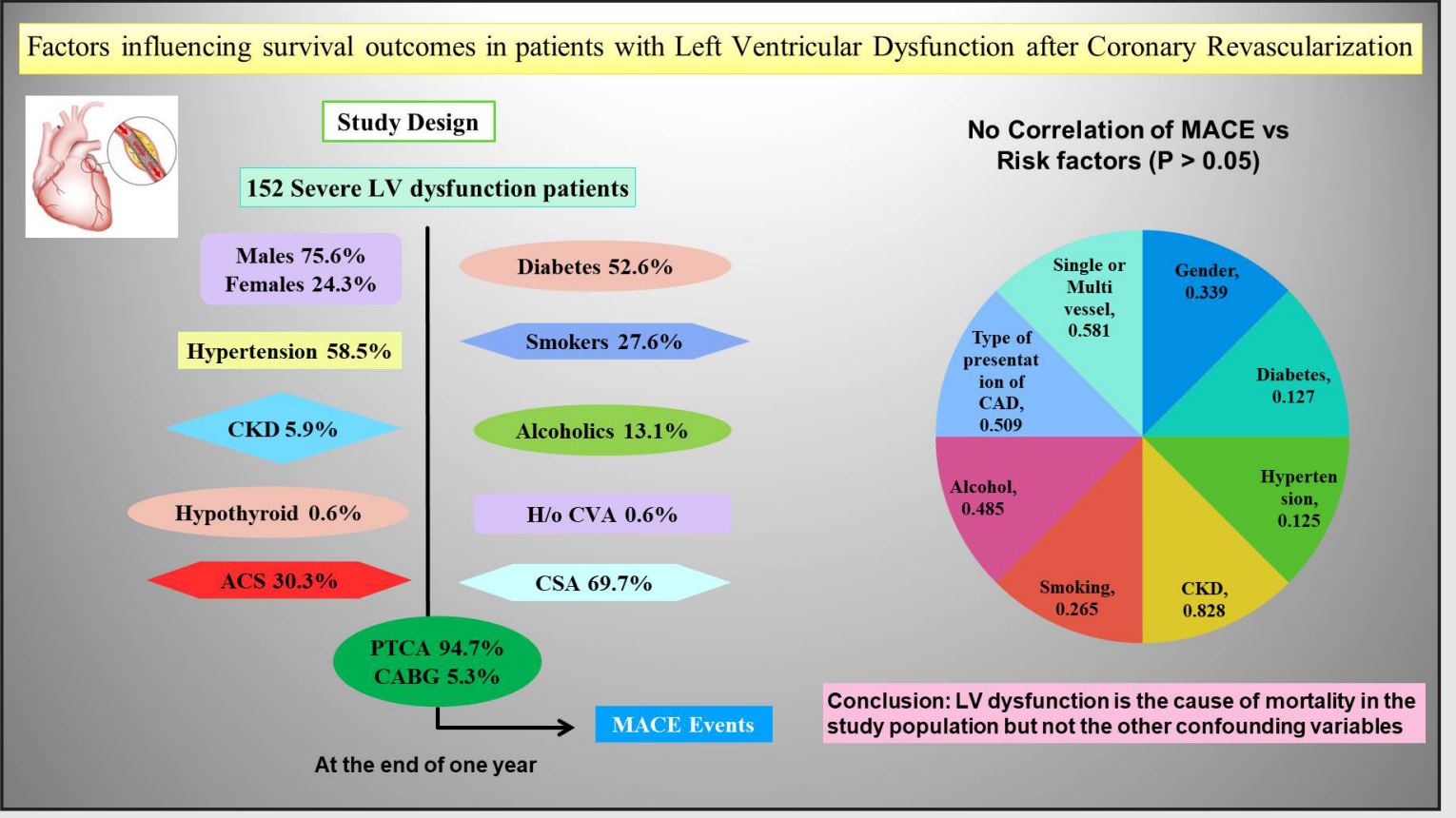

\section{Introduction}

Outcomes in patients with left ventricular (LV) dysfunction after coronary revascularization are influenced by multiple factors. Myocardial stunning and hibernation can occur as a result of LV dysfunction, mostly ischemic in nature. Several hours after myocardial ischemia, contractile dysfunction persists, but then full functional recovery occurs, which may probably be due to myocardial stunning. ${ }^{1}$ Percutaneous coronary intervention (PCI) and stenting gives symptomatic relief and improvement in outcomes because of LV remodeling reversal. Concept of the viable myocardium led to the selection of patients with LV dysfunction, which helps in patient selection for coronary revascularization. This aids in categorizing patients into LV dysfunction with scarred myocardium and LV dysfunction with ischemic but viable myocardium. ${ }^{2}$ If imaging studies are performed, after revascularization, areas that are associated with functional recovery are the regions that exhibit a reduction in both myocardial blood flow and fluorodeoxyglucose (FDG) uptake (scarred myocardium). Areas with intact FDG uptake or enhanced uptake, despite poor myocardial perfusion, are usually due to hibernating myocardium. ${ }^{3}$ Thus revascularization in severe LV dysfunction patient may be of benefit and may have implications on the outcome. ${ }^{4}$

So, through this study, we attempted to observe the influence of risk factors on outcomes in severe LV dysfunction patients after revascularization and delineate if any of them have an effect on one-year mortality of such patients.

\section{Aim \\ To study the impact of various modifiable and non- modifiable risk factors on outcomes after coronary artery revascularization in patients with severe LV dysfunction.}

\section{Objectives}

To study the impact of various risk factors like gender, age, type 2 diabetes mellitus (DM), hypertension (HTN), chronic kidney disease (CKD), cerebrovascular accident (CVA), obesity, hypothyroidism, and smoking and alcoholism on one-year major adverse cardiovascular events (MACE), which included heart failure, reinfarction (nonfatal), recurrence of angina, and repeat $\mathrm{PCI}$ or coronary artery bypass grafting (CABG). 


\section{Material and Methods}

This is a prospective single-center cohort study done at a tertiary care center for cardiology.

This study was a time-bound study done from January 2017 to December 2018 and all consecutive patients with severe LV dysfunction, who underwent coronary revascularization, were included in the study. As many as 152 patients with severe $\mathrm{LV}$ dysfunction $(\mathrm{EF}<35 \%)$ were enrolled in the study.

\section{Data Collection}

After obtaining institutional ethics committee approval, the study sample collection was started in January 2017. Written and informed consent in the local language was obtained from all eligible participants after explaining the objectives and the nature of the study. All the patients with severe LV dysfunction belonging to ST elevation myocardial infarction (STEMI), non-ST elevation myocardial infarction (NSTEMI), unstable angina, and silent myocardial ischemia were included in the study. In patients with LV dysfunction, which is of more than 7 days duration, myocardial viability was assessed by perfusion scan and/or echocardiography, and patients with significant viable myocardium were taken up for coronary revascularization. After coronary revascularization by percutaneous transluminal coronary angioplasty (PTCA) with drug-eluting stents or CABG, all patients were started on dual antiplatelet drugs, statins, $\beta$-blockers, angiotensin-converting-enzyme (ACE) inhibitors, loop diuretics, and mineralocorticoid receptor antagonists, as per the American Heart Association guidelines.

Patients were followed prospectively for one year and at the end of one year, impact of sex, age, HTN, type 2 DM, CKD, obesity, hypothyroidism, CVA, and smoking and alcohol on one-year MACE, which included heart failure, reinfarction (nonfatal), recurrence of angina and repeat PCI or CABG, were analyzed and statistically approached to observe the impact of those risk factors on one-year mortality and determine whether the timing and mode of revascularization, and number of coronary arteries involved, had any influence on mortality or MACE events. Comparing different parameters of the study with respect to mortality a regression analysis was made at the end of one year.

\section{Results}

As many as 152 patients of severe LV dysfunction ( $E F<35 \%$ ) were included in the study, of which 115 (75.6\%) were males, and 37 were females (24.3\%), with a mean age of 57.6 years at the time of presentation (-Fig. 1).

Eighty-nine (58.5\%) patients were hypertensive, 80 (52.6\%) patients were diabetic, 42 (27.6\%) patients were smokers, $20(13.1 \%)$ patients were alcoholics, nine (5.9\%) patients had CKD, one $(0.6 \%)$ patient had hypothyroidism, and one $(0.6 \%)$ patient had a history of CVA. (-Fig. 2 ).

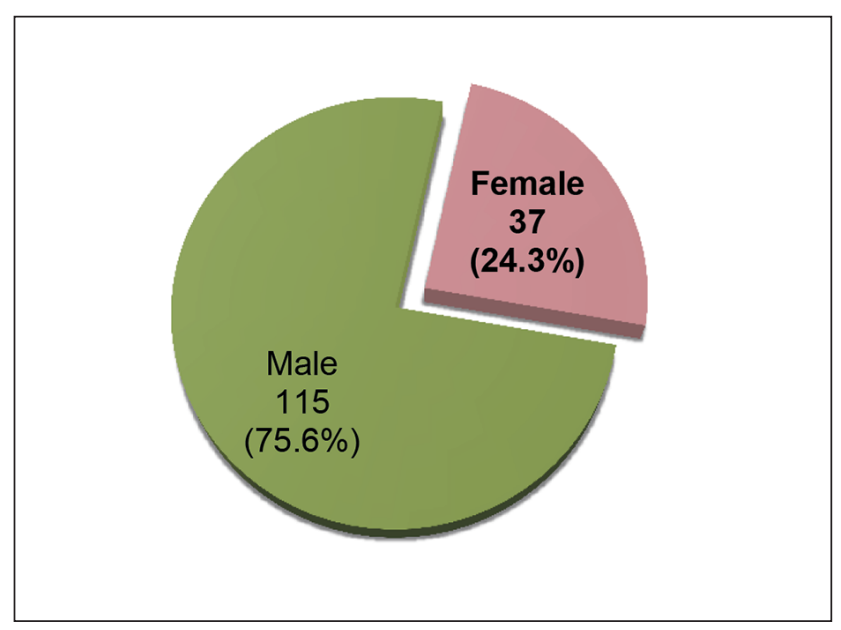

Fig. 1 Gender distribution.

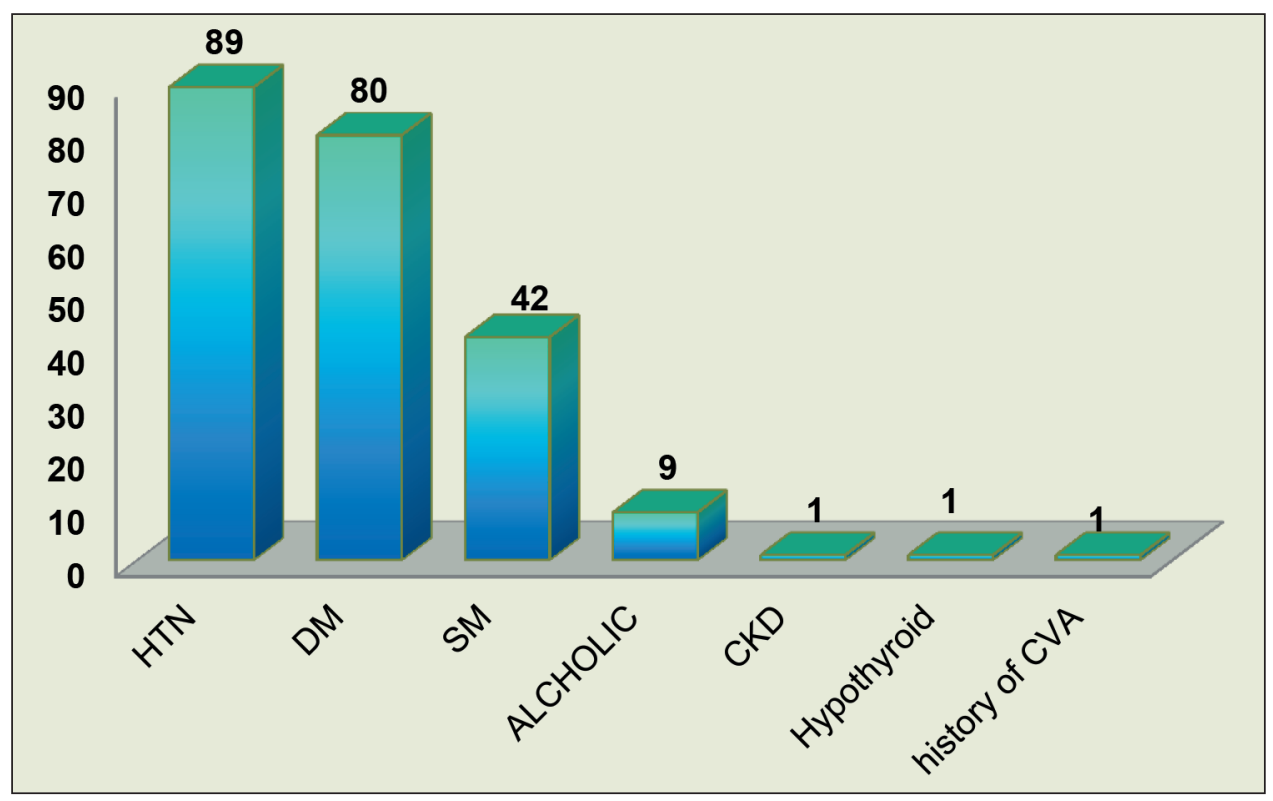

Fig. 2 Distribution of various parameters in study. 
Forty-six (30.2\%) patients presented with acute coronary syndrome (ACS), and $106(69.7 \%)$ patients presented with chronic stable angina (CSA) (- Fig. 3).

As many as 144 (94.7\%) patients underwent PTCA, while 8 (5.2\%) patients had undergone CABG (-Fig. 4).

At the end of one year, mortality was six patients (3.94\%). At the end of one-year, regression analysis was done for all the confounding variables by observing their influence on the MACE or mortality, and none of them showed statistically significant influence $(p>0.05)$.

Upon performing the chi-square test for follow-up patients, outcomes versus age, sex, HTN, DM, smoking, alcohol, type of coronary artery disease (CAD), single or multivessel disease and abnormal glomerular filtrate rate (GFR), it was observed that the $p$-value was not significant for any of the parameters, which imply that LV dysfunction in itself was the intrinsic predictor of outcomes among follow-up patients. Similar results were observed in performing a chi-square test for mortality versus clinical parameters. The findings are elucidated in

- Table 1.

When demographic, laboratory parameters were compared between males and females, none of the parameters had statistical significance except that females had a more severe stage of chronic kidney disease than males with statistical significance. When the type of intervention was compared between males and females, CABG was done in eight patients (5.2\%) who were exclusively males and the difference was statistically significant (- Table 2 ).

When outcomes (both in hospital and at one-year follow-up) were compared between males and females, there was no significant difference between males and females in terms of in hospital complications ( $p$-value 0.97 ) and at one-year follow-up ( $p$-value 0.55) (-Table 3).

Also, the parameters were statistically approached with respect to MACE. It had yielded similar results; however, statistical significance was observed between the occurrence of MACE and type of CAD ( $p$-value 0.028).

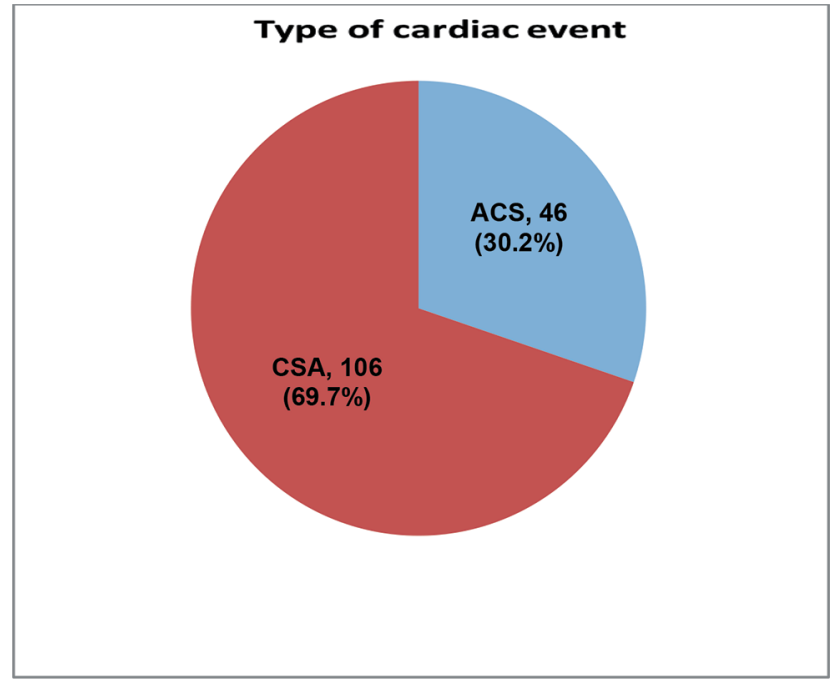

Fig. 3 Type of cardiac event at presentation.

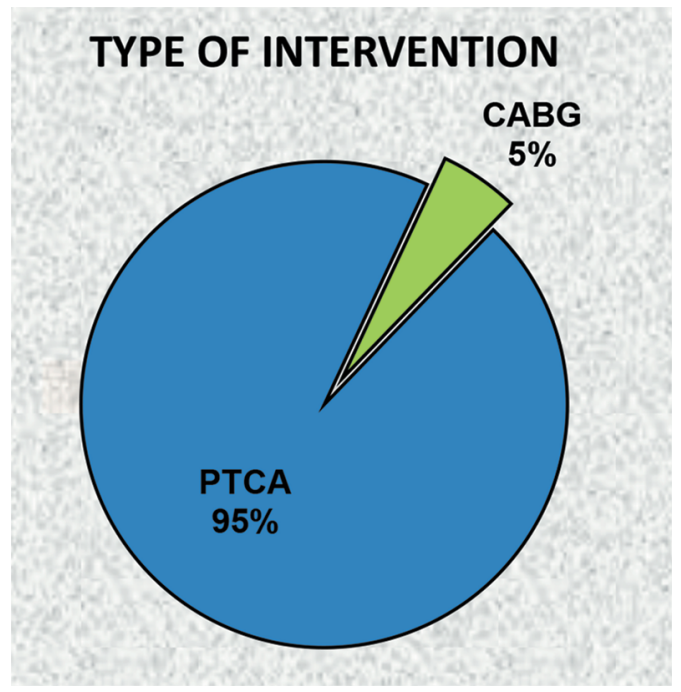

Fig. 4 Showing type of intervention in the study.

Table 1 Binary logistic regression: mortality versus clinical parameters

\begin{tabular}{|l|l|l|l|l|l|}
\hline Source & DF & Adj dev & Adj mean & Chi-square & $p$-value \\
\hline Regression & 9 & 12.0712 & 1.34124 & 12.07 & 0.209 \\
\hline Age & 1 & 2.3941 & 2.39411 & 2.39 & 0.122 \\
\hline Sex & 1 & 0.9144 & 0.91439 & 0.91 & 0.339 \\
\hline HTN & 1 & 2.3567 & 2.35672 & 2.36 & 0.125 \\
\hline DM & 1 & 2.3238 & 2.32375 & 2.32 & 0.127 \\
\hline Smoking & 1 & 1.2436 & 1.24362 & 1.24 & 0.265 \\
\hline Alcohol & 1 & 0.488 & 0.48803 & 0.49 & 0.485 \\
\hline Type of presentation of CAD & 1 & 0.4365 & 0.43655 & 0.44 & 0.509 \\
\hline Single or multivessel disease & 1 & 0.3049 & 0.30486 & 0.3 & 0.581 \\
\hline GFR abnormal & 1 & 0.0473 & 0.04734 & 0.05 & 0.828 \\
\hline
\end{tabular}

Abbreviation: CAD, coronary artery disease; GFR, glomerular filtration rate; HTN, hypertension. 
Table 2 Comparison of demographic, laboratory and parameters comparing males and females

\begin{tabular}{|c|c|c|c|}
\hline Variable & Males $(n=115)$ & $\begin{array}{l}\text { Female }(n= \\
37)\end{array}$ & $p$-value \\
\hline Age (yrs.) & $57.20 \pm 11.47$ & $58.86 \pm 10.71$ & 0.423 \\
\hline HTN & $63(54.8 \%)$ & $26(70.3 \%)$ & 0.079 \\
\hline DM & $56(48.6 \%)$ & $24(64.8 \%)$ & 0.076 \\
\hline SM & 41 (35.7\%) & $1(2.7 \%)$ & 0.000 \\
\hline Alcoholics & $20(17.3 \%)$ & 0 & - \\
\hline $\mathrm{FH}$ of $\mathrm{CAD}$ & 0 & 0 & - \\
\hline GFR & $78.80 \pm 48.82$ & $63.77 \pm 39.62$ & 0.062 \\
\hline Hemoglobin (gm. \%) & $12.78 \pm 2.05$ & $11.45 \pm 1.77$ & 0.000 \\
\hline CSA & $32(27.8 \%)$ & $14(37.8 \%)$ & 0.266 \\
\hline Type of ACS-MI & 25 (78.1\%) & $9(64.2 \%)$ & 0.348 \\
\hline CKD & 87 (75.6\%) & $29(78.4 \%)$ & 0.729 \\
\hline \multicolumn{4}{|l|}{ Stage of CKD } \\
\hline Stage 1 & $1(1.1 \%)$ & 0 & 0.315 \\
\hline Stage 2 & $40(45.9 \%)$ & $8(27.5 \%)$ & 0.062 \\
\hline Stage $3 \mathrm{~A}$ & $23(26.4 \%)$ & $6(20.7 \%)$ & 0.518 \\
\hline Stage 3B & $21(21.1 \%)$ & $9(31.03 \%)$ & 0.479 \\
\hline Stage 4 & $2(2.29 \%)$ & $6(20.7 \%)$ & 0.017 \\
\hline PCI previous cardiac procedure & $21(18.2 \%)$ & $4(10.8 \%)$ & 0.233 \\
\hline CABG & $8(6.9 \%)$ & 0 & $0.003^{*}$ \\
\hline SVD & $79(68.9 \%)$ & $26(70.3 \%)$ & 0.856 \\
\hline Route of procedure-radial & $103(89.5 \%)$ & $30(81.08 \%)$ & 0.228 \\
\hline Stent size & $2.94 \pm 0.32$ & $2.96 \pm 0.34$ & 0.753 \\
\hline Stent length & $21.54 \pm 7.51$ & $21.32 \pm 8.82$ & 0.892 \\
\hline In hospital complication & $3(2.6 \%)$ & $1(2.7 \%)$ & 0.975 \\
\hline One-year follow-up & $14(12.17 \%)$ & $6(16.2 \%)$ & 0.551 \\
\hline
\end{tabular}

Abbreviations: ACS, acute coronary syndrome; CABG, coronary artery bypass grafting; CAD, coronary artery disease; CKD, chronic kidney disease; CSA, chronic stable angina; DM, diabetes mellitus; GFR, glomerular filtration rate; FH, familial hypercholesterolemia; HTN, hypertension; MI, myocardial infarction; $\mathrm{PCl}$, percutaneous coronary intervention; SVD, single vessel disease.

*Severe TVD requiring CABG was seen only in males with statistical significance.

Table 3 Comparing the outcomes (both in hospital and one-year follow-up) between males and females

\begin{tabular}{|l|l|l|l|}
\hline Variables & Males $(\boldsymbol{n}=115)$ & Female $(\boldsymbol{n}=37)$ & $\boldsymbol{p}$-value \\
\hline In hospital complications & $3(2.6 \%)$ & $1(2.7 \%)$ & 0.975 \\
\hline One-year follow-up & $14(12.17 \%)$ & $6(16.2 \%)$ & 0.551 \\
\hline
\end{tabular}

\section{Discussion}

CAD is one of the main causes of morbidity and mortality worldwide. LV systolic function has prognostic value in CAD, so assessment of left ventricular ejection fraction (LVEF) should be done in all patients after acute myocardial infarction (AMI). Although reperfusion therapy has been found effective in the reduction of complications of AMI, LVEF impairment is a common consequence of an acute coronary event.

A meta-analysis of 19 studies was performed with a sample size of 4766, in which PCI among patients with LV dysfunction (EF < 40\%) and one-year mortality were studied. LV dysfunction patients who underwent PCI had acceptable one-year mortality. ${ }^{5}$ This is similar to the findings of our study results.
Several studies have compared PCI versus CABG and found that $\mathrm{PCI}$ is a noninferior mode of revascularization, ${ }^{6}$ which is similar to our study result. Even though studies like BARI2D have highlighted the role of diabetic control in the prevention of MACE in post-PCI patients, our study did not show a statistically significant difference in outcome with respect to DM and MACE in severe LV dysfunction, ${ }^{7}$ suggesting that DM has no impact on outcomes in patients with severe LV dysfunction.

Studies showed low eGFR affecting MACE rates in severe LV dysfunction patients undergoing PCI, but our study did not find a statistical significance of low e GFR with outcomes. ${ }^{8}$

Studies have shown no effect of smoking, alcohol, age, sex, HTN, and DM in relation to MACE in postrevascularization patients, ${ }^{9}$ which is consistent with our findings. 


\section{Limitation}

Individual variability in modifiable risk factors and their severity were not compared, and it might be one of the confounding factors.

\section{Conclusion}

From our prospective follow-up cohort study conducted to observe the influence of various parameters on the outcomes of mortality or MACE events in patients with severe LV dysfunction, after revascularization at the end of one year, no significant relationship could be determined between the MACE or mortality and gender, age, HTN, type II DM, CKD, smoking or alcohol. Severe CAD requiring CABG was seen only in males. There was no statistically significant correlation between in-hospital complications or MACE events and males or females at one year of follow-up.

On the other hand, MACE or mortality may be attributed directly to LV dysfunction itself. The association of severe LV dysfunction to one-year mortality was found to be the most influential factor after adjusting for all confounding variables, either single or combined, after applying binomial regression analysis.

\section{Audio}

Audio file for this article is available at https://doi.org/ $10.1055 / \mathrm{s}-0041-1727566$.

\section{Conflict of Interest}

None declared.

\section{References}

1 Camici PG, Prasad SK, Rimoldi OE. Stunning, hibernation, and assessment of myocardial viability. Circulation 2008;117(1):103-114

2 Buckley O, Di Carli M. Predicting benefit from revascularization in patients with ischemic heart failure: imaging of myocardial ischemia and viability. Circulation 2011;123(4):444-450

3 Auerbach MA, Schöder H, Hoh C, et al. Prevalence of myocardial viability as detected by positron emission tomography in patients with ischemic cardiomyopathy. Circulation 1999;99(22):2921-2926

4 Yanagawa B, Lee J, Puskas JD, Verma S. Revascularization in left ventricular dysfunction: an update. Curr Opin Cardiol 2019;34(5):536-542

5 Kunadian V, Pugh A, Zaman AG, Qiu W. Percutaneous coronary intervention among patients with left ventricular systolic dysfunction: a review and meta-analysis of 19 clinical studies. Coron Artery Dis 2012;23(7):469-479

6 Kunadian V,Zaman A, Qiu W. Revascularization among patients with severe left ventricular dysfunction: a meta-analysis of observational studies. Eur J Heart Fail 2011;13(7):773-784

7 BARI 2D Study Group. Frye RL, August P, Brooks MM, et al. A randomized trial of therapies for type 2 diabetes and coronary artery disease. N Engl J Med 2009;360(24):2503-2515

8 Hsu CC, Lu YC, Chiu CA, et al. Levels of indoxyl sulfate are associated with severity of coronary atherosclerosis. Clin Invest Med 2013;36(1):E42-E49

9 Tsai IT, Wang CP, Lu YC, et al. The burden of major adverse cardiac events in patients with coronary artery disease. BMC Cardiovasc Disord 2017;17(1):1PubMed 